\title{
La forza della vulnerabilità. Il mediatore come performer al confine tra culture diverse
}

The strength of the vulnerability. The broker as a performer on the border between the different cultures

\section{Stefano De Matteis}

\section{OpenEdition}

\section{Journals}

Edizione digitale

URL: http://journals.openedition.org/aam/1303

DOI: $10.4000 /$ aam. 1303

ISSN: 2038-3215

\section{Editore}

Dipartimento Culture e Società - Università di Palermo

Notizia bibliografica digitale

Stefano De Matteis, «La forza della vulnerabilità. II mediatore come performer al confine tra culture diverse », Archivio antropologico mediterraneo [Online], Anno XXII, n. 21 (1) | 2019, online dal 30 juin 2019, consultato il 23 septembre 2019. URL : http://journals.openedition.org/aam/1303 ; DOI : 10.4000/aam.1303

Questo documento è stato generato automaticamente il 23 septembre 2019

Archivio antropologico mediterraneo 


\title{
La forza della vulnerabilità. Il mediatore come performer al confine tra culture diverse
}

\author{
The strength of the vulnerability. The broker as a performer on the border
}

between the different cultures

Stefano De Matteis

\section{Premessa}

1 Le considerazioni che seguono mirano a mettere a fuoco una questione essenziale: il rispetto della complessità culturale di ogni individuo. E si tratta di una cosa di cui tenere conto non tanto nel luogo proprio del suo esistere ma, soprattutto, nei processi di migrazione, movimento e spostamento, forzati o meno che siano. Dato che ci riferiremo a casi molto particolari, che sono sempre più all'ordine del giorno, mettere al centro la complessità culturale di ciascuno vuol dire affermare la necessità "politica" di interventi che non riguardino solo la ricaduta pratica di ordine sociale (come e in che modo accogliere, eventuali limiti numerici, organizzazione tecnica, ecc.), ma che comportino la capacità di immaginare strade percorribili che muovano nella considerazione delle differenze, ipotizzando soluzioni che abbiano una funzione effettiva per il dialogo e il rispetto fra le culture e questo a partire proprio dai soggetti, dalle persone considerate nella loro interezza, cioè come espressioni "incarnate" di culture varie e diverse. Significa inoltre affermare il compito centrale che può svolgere a tale scopo l'antropologia non solo per le sue capacità di analisi, ma anche per gli effetti materiali e "applicati" che essa può fornire nello scambio e nel contatto tra culture declinate in modo concreto.

2 Tale ragionamento sarà articolato in tre passaggi. In un'epoca di post globalizzazione come la nostra considereremo, in breve, le interpretazioni più diffuse che hanno narrato l'abbandono degli spazi locali definendo alternativamente la società come lo spazio dell'incertezza o del rischio. Utilizzeremo a titolo esemplare un caso offerto 
dalla cronaca recente. E, per il suo valore indicativo, lo metteremo a confronto - con un metodo à la Bateson - con altri due casi volutamente molto diversi tra loro. Questo ci permetterà di evidenziare ipotesi di intervento, linee generali che ci saranno utili a indicare le funzioni che potrebbero ricoprire operatori capaci di trasformarsi in agenti attivi di questi cambiamenti. Vivere e partecipare a questi processi dall'interno, permetterebbe loro di assumere l'importante ruolo di performer culturali, abili e capaci innanzitutto nel relazionarsi ai soggetti migranti, considerati non semplicemente un numero (quando non un estraneo o addirittura un nemico), ma figure complesse e culturalmente articolate con cui interagire sia nelle relazioni di base sia quando queste esprimono, in tutti i sensi, le proprie difficoltà. Tutto ciò permetterebbe al performer di svolgere un ruolo centrale e non solo tecnico e organizzativo, nella società dell'incertezza, della paura e dei rischi.

\section{Globalizzazione: disgrazie, incertezze, rischi ${ }^{1}$}

3 La globalizzazione è stata, e in parte lo è ancora, una trasformazione di carattere essenzialmente economico e finanziario che ha esaltato numerosi luoghi comuni e rafforzato molte delle tendenze che erano caratteristiche della precedente postmodernità. E, nello stesso momento, ha cementato fedi esistenti e strutturato credenze inedite ${ }^{2}$. Tra queste, la più diffusa è quella a riguardo di un mondo divenuto globale che ha oramai superato definitivamente le localizzazioni abbattendone $\mathrm{i}$ confini ${ }^{3}$. Non è un caso che la globalizzazione venga definita, da Anthony Giddens, come «l'intensificazione di relazioni sociali mondiali che collegano tra loro località distanti facendo sì che eventi locali vengano modellati dagli eventi che si verificano a migliaia di chilometri di distanza e viceversa» (Giddens 1994: 71).

4 Se in un passato ancora recente il progresso industriale aveva creato un movimento che dalle comunità locali - piccole o medie - spingeva verso aperture regionali o nazionali (cfr. Gellner 1986), l'attualità si configura invece senza limiti e offre la fantasia del superamento di ogni tipo di barriera locale, geografica o sociale: a partire dall'ultimo decennio del secolo scorso la parola d'ordine è globale. Ma di questo eventuale abbandono degli universi locali grazie a uno spostamento - quando non a una fuga verso il cuore occidentale di una globalità tutta da capire e definire, si sono esaltate le possibilità implicite che esso offre ${ }^{4}$, mentre sono stati valutati poco o nulla i limiti e i pericoli. Da questo punto di vista è stata forse Mary Douglas, in un commento particolarmente significativo, una delle prime a metterci sull'avviso dei possibili rischi quasi impliciti che questi cambiamenti portano con loro:

Uscire dalla comunità locale significa sconfiggere la sua tirannia. Il fuggiasco è spesso felice di scrollarsi di dosso le sue fastidiose costrizioni, e non dà peso alle sue vecchie compensazioni. Aggiungerei che la liberazione dalle piccole comunità significa anche la perdita delle vecchie protezioni. I mercati ci inducono (deliberatamente) a sollevarci dal torpore delle nostre confortevoli nicchie locali e ci trasformano in attori liberi da ogni impedimento, mobili in un sistema mondiale. Ma rendendoci liberi ci lasciano esposti. Ci sentiamo vulnerabili (Douglas 1996: 33).

Ovviamente nessuno ha mai sostenuto che le comunità locali fossero indenni da ogni forma di pericoli e di rischi, provenienti dall'interno stesso o dall'esterno, solo che a questi, guardati dal di dentro, veniva dato un altro nome, si chiamavano disgrazie. $\mathrm{E}$, in quanto tali, arrivavano senza preavviso, come la tegola inaspettata che ti cade sulla testa. Sappiamo bene che ogni avvenimento apparentemente inspiegabile ha bisogno di 
una spiegazione e che questa dev'essere in qualche modo razionale: una tegola non si stacca da sola; quindi c'era chi individuava in quella "disgrazia" l'intervento umano, nel senso immediato e diretto di qualcuno che l'ha staccata e l'ha tirata, o in modo indiretto di chi faceva ricorso a qualche forma di stregoneria ${ }^{5}$. La sociologia, la più "razionale" delle scienze sociali, ci insegna d'altro canto che esistono ulteriori pratiche di controllo: se quel tetto fosse stato sottoposto alle giuste regole di conservazione, sicuramente la tegola non si sarebbe staccata. Ma, indipendentemente dalla necessaria manutenzione e in assenza di un franco tiratore o di una forza magica, rimane un ampio spazio che dobbiamo comunque lasciare al caso: nonostante le possibili ragioni che possiamo tirare in ballo, resta il fatto che, in quello specifico momento, ci fosse proprio quella determinata persona a passare di lì e ad essere colpita. Da una tegola.

6 Il mondo tradizionale - il mondo fino a ieri, come vedremo - era attrezzato ad affrontare le disgrazie: indipendentemente dalle spiegazioni da trovare quando esse colpiscono, nella gran parte dei casi si cercava di prevenirle con un sapere messo in gioco "preventivamente", che permetteva di evitare, per come possibile, le disgrazie. Un sapere in quanto tale, che vive di comunicazione e si alimenta di conoscenze condivise grazie alla parola. Si trattava di costruire e partecipare saperi che fossero capaci non solo di contenere quella vulnerabilità, ma di evitare i rischi possibili cui si poteva andare incontro, qui come altrove. Su questo la letteratura etnoantropologica offre un panorama ampio e variegato di molte delle forme di "protezione" locali che vengono messe in atto ${ }^{6}$.

7 Ovviamente, per ritornare alla Douglas, ciò che va tenuto presente non è il dato positivo che riguarda l'abbandono delle "tirannie" locali, e neanche la perdita delle "vecchie protezioni», quanto il fatto che trasformarsi in «attori liberi da ogni impedimento", l'esaltazione delle possibilità per la fuoriuscita verso un mondo aperto $\mathrm{e}$ per certi versi sconosciuto, espone a una condizione di vulnerabilità. Questo tema lo ritroviamo come centrale in un processo ancor più generale, cioè fin da quando $i$ cambiamenti sociali hanno reso "l'uomo flessibile», un traguardo raggiunto grazie all'abbandono di molte certezze e costruendo quella che sarà chiamata la società dell'incertezza (Bauman 1999). E questo anche dando al termine un valore positivo come, ad esempio, nei manuali di gestione aziendale i quali ci informano che vivere in un continuo stato di vulnerabilità serve a esaltare la necessità dell'assunzione dei rischi. Riferendosi proprio a questa situazione di flessibilità si arriva a parlare del rischio declinato sempre più come una «necessità quotidiana di massa» (cfr. Sennet 1999: 82, 79).

8 Siamo lontani dai tempi in cui Julien Sorel o Vautrin evolvevano narrativamente proprio decidendo di correre dei rischi e, mettendo in gioco quanto avevano, si trasformavano così in figure quasi eroiche. Ma siamo lontani anche dai tempi in cui correre dei rischi era un «territorio esclusivo dei capitalisti d'assalto o degli individui straordinariamente avventurosi»(ivi: 79): nella modernità avanzata, la produzione sociale di ricchezza «si accompagna sistematicamente alla produzione sociale di rischi» (Beck 2000: 28). Tanto che lo stesso Ulrich Beck, in una sorta di "crescendo", ha disegnato la società contemporanea come la «società mondiale del rischio»7.

9 Per quanto riguarda l'antropologia, fin dagli anni Ottanta del Novecento, Douglas ha cercato di individuare e isolare i concetti di pericolo e rischio in modo da trovare per quest'ultimo i collegamenti tra comportamenti individuali, istituzioni sociali e pratiche politiche (Douglas, Wildavsky 1982; Douglas 1991). Da allora gli studi sul rischio hanno 
avuto uno sviluppo esteso e una diffusione capillare ${ }^{8}$, e questo forse perché l'idea di rischio, con «la sua terminologia universalizzante, la sua astrattezza, la sua forza di sintesi, la sua scientificità, la sua connessione con l'analisi oggettiva la rende perfetta» (ivi: 33). Infatti, il termine rischio viene sempre più inteso in un'accezione tanto ampia da coprire una casistica fin troppo estesa, al punto da riguardare (anche) le possibilità a quanto pare spesso molto prossime - di perdere le certezze acquisite e cadere, scendere, nella scala sociale. In questi anni sono cambiate le generali prospettive di vita e hanno preso corpo delle trasformazioni che espongono tutti a un movimento sociale inatteso. In un saggio del 1997 (tradotto in Italia significativamente proprio nel 2008), Ulrich Beck precisa che

a ridurre le persone in povertà non è più solo la disoccupazione, ma sono anche eventi "normali" come, per esempio, un divorzio, una malattia improvvisa o uno sfratto (Beck 2008: 60).

E lo stesso autore continua interrogandosi sulle possibilità di ognuno di costruire la propria vita, cercando di evidenziare il punto in cui inizia una biografia a rischio:

Potersi dedicare a costruire la propria vita non necessita forse innanzitutto di disporre di denaro proprio, di una professione propria, di un proprio spazio, nonché della sicurezza fondamentale di non venire aggrediti o rapinati mentre si cammina per strada? (Beck 2008: 58)

Denaro, professione, spazio, sicurezza. Ecco individuati quattro elementi che costituiscono gli elementi essenziali della vita sicura (almeno secondo Beck) ${ }^{9}$. Maneggiando queste argomentazioni cui facciamo riferimento per tracciare le linee generali di un iniziale panorama interpretativo, ci si rende conto che stiamo sintetizzando un ragionamento che ha una forte radice "europea", espressione del pensiero occidentale (soprattutto di formazione americana e tedesca) e che muove dai possibili cambiamenti che riguardano le vite individuali una volta che sono venuti meno i rassicuranti appoggi tradizionali e le sicurezze derivate dalle condizioni e dai privilegi propri delle società del benessere occidentali.

Proviamo ora a uscire da una logica occidentale e cerchiamo di verificare se tutte queste considerazioni reggono agli scenari contemporanei.

\section{Milano, Niguarda}

13 Come da partitura, propongo ora di esaminare un recente caso che ho in parte ricostruito e che servirà da campione per lo snodo delle nostre argomentazioni per poi passare ad altri casi, più classici e lontani tra loro nel tempo e nello spazio, in modo da poter elaborare i nostri ragionamenti e costruire un quadro generale grazie a delle prospettive molto diverse da quelle cui abbiamo fatto rifermento finora.

Trasferiamoci a Milano. Al Quartiere Niguarda. È l'alba dell'11 maggio 2013. Adam Mada Kabobo è un ghanese che ha passato la notte in un cantiere. Si aggira per le strade armato di piccone. «Nella torpidezza del risveglio aveva preso con sé un piccone con l'idea di farsi giustizia» (Dell'Acqua, D'Autilia 2017: 47). Colpisce le prime persone che incontra. Muoiono in tre. Adam Mada Kabobo è arrivato in Italia con un barcone nel luglio del 2012. Viene dal Ghana. È "ospitato" in un centro di prima accoglienza e qui con altri giovani immigrati organizza una protesta. Per questo viene incarcerato a Lecce. È definito psicotico. Riceve l'obbligo di dimora nel centro accoglienza richiedenti asilo (Cara) di Foggia da cui fugge per arrivare a Milano il 16 aprile. Qui è intercettato e 
controllato dalle forze dell'ordine. Ma non può far altro che bivaccare e restare sempre più isolato. E questo anche per un altro motivo, sostanziale: Kabobo parla solo un dialetto ghanese che nemmeno i mediatori culturali capiscono. Quando viene arrestato dopo gli omicidi, pronuncia solo poche parole sconnesse legate al cibo e alla fame. E successivamente dirà di aver compiuto gli omicidi perché sentiva delle voci che glielo ordinavano ${ }^{10}$.

Il resoconto della perizia tiene conto di questo dato, ma non risulta sufficiente: «Dice che le voci gli suggerivano di colpire, ma che aveva anche motivazioni non psicologiche. Abbiamo anche cercato di capire se c'era l'intervento di qualche credenza particolare. Ma non c'era, lo dice lui stesso». L'accusa formula una richiesta di ergastolo, ridotta per seminfermità a venti anni di detenzione cui seguiranno altri tre anni di misura di sicurezza detentiva; in parole povere, sarà trasferito all'ospedale psichiatrico giudiziario ${ }^{11}$. Per i colloqui era stata individuata una mediatrice culturaletraduttrice che ha permesso ai periti di affermare che «sull'intendere, diciamo che è poco attrezzato, è possibile che abbia una scarsa dotazione intellettiva» ${ }^{12}$.

Naturalmente ci troviamo davanti a un caso dove è forte il potere dei simboli e della loro efficacia a più livelli e in varie direzioni: innanzitutto lo "straniero", un estraneo la cui alterità comincia dal colore della pelle, un "diverso" anche per come si presenta, dall'abito dimesso, liso e consumato di un poveraccio. Da questo punto di vista bisogna ricordare che la società dell'incertezza non inculca solo il brivido che prende corpo nel rischio di cadere verso il basso della scala sociale, ma questo porta con sé, impliciti, due risvolti contrapposti: la paura della diversità in genere, che diventa ancora più accentuata nei riguardi della povertà e di tutti i suoi simboli come aporofobia generalizzata e, nello stesso tempo, la costruzione dell'immagine di sé come modello perfetto per gli altri.

17 Oltre al fatto di non essere europeo, è anche un ribelle, perché aveva protestato per i propri diritti e per questo era stato ridotto in stato di detenzione, cui era seguita una prima diagnosi di psicosi. Ma soprattutto, Kabobo viveva nella quasi totale assenza di comunicazione linguistica, in quanto parlava un dialetto che nessuno conosceva.

18 Ora, al di là dell'efferato delitto, sul quale non spetta a noi esprimere giudizi, il profilo di Kabobo si presenta perfetto per la sintesi di problemi e di questioni che solleva e a cui rimanda a livello antropologico e sociale. Qui ne accenneremo perché ci servirà come modello di riferimento per le comparazioni che andremo a fare successivamente e, soprattutto, per le considerazioni finali.

19 Partendo dal presupposto che non conosciamo la sua traiettoria biografica profonda, proviamo a evidenziare la catena dei rischi che vengono messi in campo in questo caso: 1. per costruirsi una vita, Kabobo cerca di evitare il rischio di vivere nel proprio paese; questo comporta che deve assumersi il rischio di una traversata su di un barcone; per raggiungere poi un paese a lui sconosciuto e di cui forse non sa nulla. E già solo questo apre a scenari molteplici: dall'abbandono delle condizioni "locali", all'interrogarsi sull'eventuale scelta non-scelta del luogo di arrivo, l'organizzazione e i modi in cui tutto questo è stato possibile, fino alla scelta, se di scelta si può parlare, di muovere verso un universo ignoto, cui segue ovviamente l'impatto con la nuova realtà sociale e culturale di cui non sa;

2. una volta arrivato e condannato per ribellione, viene segregato;

3. Kabobo presenta sintomi di psicosi: non sappiamo se questa risalga all'infanzia, oppure sia da correlare al rischio dettato dall'abbandono della situazione "locale" di 
partenza, oppure dipenda dalle condizioni di vita una volta raggiunto il paese ospite; 4. infine, last but not least, credo che nessuno possa immaginare cosa significhi vivere in un paese straniero, non solo da emarginato e da carcerato ma, come se non bastasse, anche da sordomuto, in quanto non conosceva la lingua e quindi non poteva parlare, discutere, chiacchierare con alcuno. Non poteva conoscere e farsi conoscere.

Dall'altra parte c'è la disgrazia, la tragedia di alcune persone che recandosi al lavoro un mattino si trovano davanti un uomo. Che li colpisce. E li uccide. Come una tegola caduta inaspettatamente. Il mondo locale è uguale quasi dappertutto, almeno per quanto riguarda le disgrazie.

Quanta attenzione (per non dire, come i sociologi, 'manutenzione') è stata offerta a Kabobo perché non arrivasse a tanto? Quanto è stato considerato come persona? E quanto è stato fatto per lui in quanto persona malata, visto che gli è stata diagnosticata una psicosi? La prospettiva che stiamo adottando ci permette di smascherare uno strabismo che per comodità (anche politica) viene esaltato: il rischio lo si personalizza, lo si incarna nel migrante, nel soggetto, nel "diverso" in tutti i sensi, dal colore della pelle, allo status di povero, alla lingua, al pantheon di credenze cui fa riferimento. Mentre in realtà è esattamente il contrario: il rischio è l'abbandono, vivere nella mancanza di attenzione e di "cure" adeguate, in quanto non bastano centri di accoglienza come prigioni, reclusioni, solo per sottrarre alla vista e per operare una cancellazione dall'attenzione generale ${ }^{13}$.

Come sostiene Ulrich Beck, tutti abbiamo bisogno «della sicurezza fondamentale di non venire aggrediti o rapinati mentre si cammina per strada», ma questo può essere in parte anche previsto, se ci fossero alcune basilari prevenzioni che riguardano sempre la cura, non poliziesca, ma delle persone che transitano e che chiedono soccorso. Persone. Sane o malate che siano.

\section{Le società tradizionali}

Trasferiamoci molto lontano, andiamo in Nuova Guinea. Ci stabiliamo momentaneamente qui per un motivo particolare: i guineani hanno elaborato numerose pratiche per «limitare il rischio». Infatti per contenerlo mettono in atto una sorta di metodo che

si compendia in questa semplice norma: se una certa azione implica ogni volta un rischio contenuto ma è necessario compierla spesso, è meglio agire sempre con prudenza, altrimenti si rischia di morire giovani o di restare invalidi (Diamond 2013: 244).

Jared Diamond ha dato un nome a questa pratica, l'ha chiamata "paranoia costruttiva». Ma tutto questo potrebbe sembrare banale se non reggesse su alcuni corollari che ci permettono degli approfondimenti mirati. Il primo riguarda il fare attenzione e il saper leggere i segnali. Diamond è in giro con la sua fidata guida, Gumini, per effettuare le sue ricerche. Si trovano in una zona vergine ed isolata. Il rischio che bande di nomadi possano arrivare per far valere il proprio diritto territoriale e dimostrare, in modi più $o$ meno violenti, di non essere proprio contenti di quella che possono reputare un'invasione è forte, anche se pare che non ci siano segnali in merito.

Nel muoversi verso la meta, Gumini all'improvviso «si fermò e si chinò a studiare con attenzione qualcosa per terra». Sembra una pianticella. Ma non lo è. «È un rametto 
conficcato per terra» sentenzia Gumini, «devono averlo staccato e messo qui a mo' di segnale» (ivi: 267).

A queste parole il nostro scienziato si sente raggelare. Per fortuna le cose vanno per il meglio. Ma

se non si presta la massima attenzione ai segnali che potrebbero rivelare la presenza degli estranei, nella società di Paia e di Gumini non si sopravvive a lungo: insospettirsi di un rametto innaturalmente conficcato nel terreno non è certo sbagliato, né passare un'ora a discuterne, né controllare che in giro non ce ne siano altri [...] meglio darsi pensiero di mille ramoscelli caduti in una posizione apparentemente non causale, piuttosto che ignorare fatalmente l'unico conficcato per terra da qualche strano esponente della razza umana (ivi: 269).

Se il rischio è uno degli elementi costanti della vita nelle società tradizionali, questo lo si contrasta con la loquacità e dedicando una grande quantità di tempo a chiacchierare e discutere dei fatti, delle cose, delle soluzioni e dei possibili rimedi ai rischi: nel caso dei guineani, «la loro conversazione è una specie di radiocronaca in diretta su tutti gli accadimenti del mattino o del giorno precedente e dà ragguagli puntuali» (ivi: 273). Naturalmente questa capacità discorsiva è quella che ci interessa e risponde a più finalità, infatti la parola mira alla condivisione e alla partecipazione, ma ha anche finalità informative e pedagogiche:

Il costante fluire della conversazione aiuta i guineani ad affrontare meglio la vita in un ambiente pericoloso come il loro. Tutto viene discusso: i minimi dettagli di ogni evento, le differenze tra ieri e oggi, ciò che potrebbe accadere in futuro, le azioni compiute da altri e le relative motivazioni. [...] Conversando senza sosta e accumulando la maggior quantità possibile di conoscenze, essi si sforzano di decifrare il mondo e si preparano ad affrontare i rischi di una vita decisamente più pericolosa della nostra (ivi: 274).

Cosa impariamo dal mondo fino a ieri e dalle società tradizionali? Una cosa fondamentale. Il caso guineano ci informa sull'importanza della parola, del dialogo, della conversazione applicata all'analisi, all'esame delle situazioni, come atto riflessivo a seguito dell'attenzione che viene data alle circostanze della vita. Ci riferiamo non solo a un sistema di osservazione ma anche a un approfondimento dialogico collettivo. Cosa ben diversa dal funzionamento di una tradizione che mette in atto una sorta di pratica "verticale" in quanto implica che ci sia qualcuno che insegna, che ha l'autorità di istruire per mezzo della parola e dell'esempio ${ }^{14}$. Ciò che ci interessa evidenziare a partire dall'esempio guineano è che parliamo di un sistema "orizzontale", di un allenamento che si può sperimentare e praticare in situazioni molto diverse tra loro, in gruppi e con persone anche di diverse culture.

Da questa prospettiva Kabobo si trovava impossibilitato a discutere, dialogare, conoscere. Nessuno poteva offrirgli il ristoro di una spiegazione o di una informazione. Il che porta a sollevare dei dubbi anche sulle iniziali diagnosi sul suo stato di salute mentale visto che in quell'occasione nessuno sapeva e poteva comunicare con lui.

Teniamo presente queste considerazioni generali che ci vengono dalle società tradizionali e proseguiamo con l'ultimo caso.

\section{Un caso lontano, ma utile}

31 A questi esempi entrambi "contemporanei", ne aggiungiamo un altro, richiamando un caso "storico", famoso e ampiamente discusso. L'etnologo Jean Rouch viene invitato da 
alcuni sacerdoti a riprendere una particolare cerimonia vudu. L'obiettivo è quello di farne un caso esemplare. Cioè far sì che quanto è accaduto divenga modello possibile, applicabile anche in altri casi. Nasce così Les Maîtres fous (Francia, 1955), che ci riporta esattamente al cuore delle questioni che stiamo intrecciando. Narro brevemente le linee principali per lasciare poi la parola a un testimone d'eccezione.

Un numero consistente di Bambara attratti dalla nascente società industriale lasciano $\mathrm{i}$ loro villaggi del medio Niger dove vivevano di agricoltura e di pesca per trasferirsi ad Accra, nella Costa d'Oro, la colonia britannica chiamata così per i giacimenti, che poi diventerà il Ghana. I migranti trovano delle condizioni materiali di vita migliori di quelle dei loro villaggi d'origine, anche se il "nuovo" mondo risulta loro estraneo in tutte le sue espressioni al punto da non poter trovare in questo alcun orizzonte di appropriazione culturale, sociale e simbolico. La condizione dei nuovi immigrati finisce per essere migliore da un punto di vista di vita materiale, ma peggiore da quello esistenziale in quanto non possono più fare ricorso ai metodi tradizionali: riti $\mathrm{e}$ cerimonie non sono più utilizzabili nel nuovo mondo, retto da nuove regole, da nuovi ideali, da un nuovo pantheon di riferimenti. Questa mancanza di difese, protezioni e strumenti cui fare ricorso li fa sentire in una sorta di vuoto culturale. E questa assenza porta molti di loro a fare i conti con l'esplosione di una serie di traumi nella loro nuova vita di migranti. Fu così che nella comunità dei Bambara a Accra affiorarono una serie di disordini psichici di tale gravità che non si riusciva a trovare modo di controllarli o di reinserirli in un definito ordine culturale. Tali disordini psichici misero in allarme le autorità in quanto neanche la medicina riusciva a trovare una soluzione efficace visto che sfuggivano a ogni tentativo di ricorso a cure "europee"15.

33 A questo punto voglio lasciare la parola a Ernesto de Martino che in più occasioni si è occupato di questioni relative alle migrazioni, alla malattia, ai cambiamenti di orizzonti culturali, e che ci offre un approfondimento specifico e molto pertinente per costruire un passaggio essenziale nella direzione in cui vogliamo procedere. Infatti, de Martino sottolinea l'importanza che uno dei bambara acquisisce, prendendo il ruolo di agente del cambiamento.

Riuscì invece a risolvere questa situazione un bambara, che era uomo di larga esperienza e aveva maggiori capacità degli altri emigranti. Costui prese alcuni elementi del vecchio dispositivo culturale - per esempio l'altare conico al centro della radura - modificandoli in funzione della nuova situazione. Divise così l'altare tradizionale in varie sezioni, la più alta delle quali ospitava il governatore come nuova divinità del pantheon industriale e coloniale, e poi via via il medico, il capo della polizia, la moglie del medico, eccetera. Alla base di questo altare conico, che rappresentava in un certo senso l'immagine mitica della situazione coloniale, vi era il magazzino delle offerte sacrificali.

In realtà l'abilità del nostro protagonista sta nel procedere a una riscrittura dei vecchi dispositivi culturali ma inserendoli dentro la nuova situazione:

Ma ciò che rendeva di particolare interesse questo riadattamento della religione tribale alla nuova situazione erano i riti e le cerimonie. I Bambara, mantenendo i vecchi riti di possessione caratteristici della loro tradizione magico-religiosa, si lasciavano ora possedere dalle divinità del nuovo pantheon. Essi erano così posseduti, nel corso delle cerimonie celebrate presso l'altare, dallo spirito del governatore inglese o dal capo della polizia o dal macchinista delle ferrovie e adoperavano come formule liturgiche le forme burocratiche che costituivano un altro elemento traumatizzante della loro nuova vita cittadina. In tal modo i traumi e i conflitti accumulati quotidianamente, e che prima esplodevano in disordini psichici veri e propri, venivano ora fatti defluire nell'ordine rituale della 
possessione e ricevevano orizzonte in figurazioni mitiche definite. Così il nuovo dispositivo culturale poté assolvere una funzione riequilibratrice e reintegratrice e i disordini psichici trovarono la loro più appropriata modalità di controllo (de Martino 1964: 225-231).

Una persona di «larga esperienza», che «aveva maggiori capacità degli altri emigranti» che evidentemente conosceva alla perfezione il funzionamento e le regole del dispositivo rituale tradizionale, riuscì a riformularlo, riscriverlo e rimodularlo applicandolo alla nuova situazione. Quest'uomo con le sue particolari doti si prese cura degli altri, si occupò di loro e dei loro problemi: se ne fece carico.

Darò un nome a questa figura, lo chiamerò performer.

\section{Il performer}

Ci riferiamo a questo termine a partire dall'accezione della sua etimologa che riguarda l'azione, chi fa, chi agisce in rapporto e in relazione agli altri, ma anche in loro aiuto e a sostegno ${ }^{16}$. Il termine ha avuto un significativo approfondimento antropologico che ci conforta a proseguire nella direzione che stiamo indicando soprattutto grazie a Victor Turner (1993 e 2014), perché ci permette di focalizzare maggiormente gli elementi che abbiamo finora individuato e evidenziato a partire dai casi etnografici con cui ci siamo confrontati. Si tratta della capacità di leggere i segnali, dell'abilità nella comunicazione, dello scambio e della diffusione della relazione dialogica. Tutti elementi che vengono finalizzati e utilizzati per inventare soluzioni ai problemi a volte inediti che si presentano, ma anche per riformulare pezzi di culture e favorire di seguito nuovi appaesamenti, per restare alla terminologia demartiniana.

Concretamente significa entrare in contatto diretto, mettere in atto la relazione fondamentale Io-Tu (sottolineata da Buber [1959] e evidenziata ancora una volta da Turner). Vuol dire avere la capacità di fare con le parole e agire con esse. Intervenire, aprire discussioni, analisi e riflessioni; condividere lo spazio dello scambio e immaginare soluzioni e alternative. Il performer è l'espressione massima di colui che fa, che stabilisce legami e struttura e ristruttura relazioni. E che, soprattutto, considera l'altro una risorsa con cui costruire assieme nel presente per il futuro.

I casi cui ci siamo rifatti, tra il mondo fino a ieri e i cambiamenti in atto, ci sono utili anche per chiarire un altro passaggio. La modernità in genere, ma soprattutto quella declinata dalla globalizzazione, ha implicita l'idea di una totale cancellazione del passato, come se questo possa scomparire con un tocco di bacchetta magica. Ma sappiamo bene che non è affatto così, in quanto ci troviamo sempre a confronto con processi di negoziazione, di meticciamento, di stratificazione (cfr. Hüsken, Neubert 2012). In questi passaggi il ruolo del performer può essere centrale nella costruzione di nuovi panorami culturali, soprattutto nella loro ricaduta concreta e materiale come quelli che nascono dalle esigenze dirette e immediate della persone. E questo anche per un altro motivo, che dobbiamo tenere sempre in evidenza in quanto credo possiamo considerarlo la questione fondamentale: parliamo di persone che vanno considerate nella loro interezza e complessità. Una delle tragedie del presente è che la cosiddetta globalizzazione ci educa a vivere, e fare i conti con, un mondo che si definisce genericamente complesso, ma non a misurarci con la prima e fondamentale complessità che è quella delle persone. 
La presa in carico dell'altro stabilisce una modulazione che è sempre più importante se teniamo assieme i due elementi della parola e dell'azione, del fare e del condividere verbalmente. Non è un caso che entrambi questi due aspetti siano le doti esclusive del performer ${ }^{17}$. In generale, in momenti di (anche apparente) cambiamento o sconvolgimento il rischio aumenta: soprattutto se non c'è alcun modo di controllare, comunicare, verificare, relazionarsi, se non c'è chi sa disporsi verso l'altro in una pratica orizzontale di partecipazione e di condivisione. E questo discorso non riguarda solo lo "straniero" o l'immigrato, ma riguarda l'altro in senso ampio e aperto, cioè tutti coloro che si trovano o esprimono un momento di particolare vulnerabilità. Ora, al di là del delitto efferato cui abbiamo fatto riferimento, la questione che solleviamo è precedente, in quanto viene molto prima delle azioni compiute: queste denunciano un'incapacità, quella di farsi carico, di prendersi cura, di permettere e favorire pratiche performative per l'invenzione di strade da frequentare e che potrebbero rivelarsi anche possibili soluzioni.

41 Eccoci arrivati così al cuore del problema. Vicini o lontani, estranei o "indigeni", bianchi o gialli, giovani o vecchi, si tratta comunque di persone che vanno considerate nella loro complessità, e questo anche per superare il limite attuale di gran parte delle istituzioni preposte, dove «mai la persona viene accolta, presa in carico per la globalità del bisogno che esprime» (Dell'Acqua, D'Autilia 2017: 47) ${ }^{18}$.

\section{Conclusioni}

42 I rischi si evitano quando li si conosce o li si immagina leggendoli "preventivamente" da segni incontrati per caso, da questa prospettiva le tecniche tradizionali ci permettono di prevenirli. Ma non solo, perché il problema nasce quando li si ignora, o li si evita, o si fa finta di nulla. Dopo non ci resta che urlare all'assassinio, seguendo logiche stereotipe agitate dalla promozione politica. Al contrario, bisogna parlarne, raccontare, comunicare e analizzare le difficoltà. Condividerle. La soluzione a eventuali rischi potrà esserci solo quando saremo disposti a condividere le nostre vulnerabilità. Grazie alla parola, al racconto, all'esempio. Altrimenti ci mettiamo nella situazione di ricevere solo tegole che cadono improvvise. E questo è valido ancor più oggi, quando nella quasi totale assenza di figure che incarnano una qualche autorità riconosciuta (nonni, genitori, insegnanti, maestri), possiamo rifarci a protagonisti che, più in generale, possiamo chiamare performer: coloro che portano attenzione all'altro, che agiscono, intervengono, si prendono cura e "inventano" soluzioni possibili e praticabili come nel caso dei Bambara di Accra.

L'esempio è importante perché ci permette alcune riflessioni ulteriori. La principale riguarda l'importanza di immaginare e preparare figure che abbiano le capacità organizzative di gestire il limite tra mondi diversi e l'agilità di muoversi al confine tra "località" altrettanto diverse: quella dei migranti e quella del luogo d'arrivo. Molto spesso le figure istituzionali dei mediatori non sono attrezzate a fare questo, non hanno questo tipo di conoscenze, qualità e competenze. La forza di questa ipotesi è nel dialogo e nella possibilità di agire leggendo i rischi e ipotizzando delle soluzioni. Il performer è un attore sociale competente e autorevole, all'interno e all'esterno, che organizza una forma di autogoverno della complessità culturale. Dove la forza meticcia della comunità si alimenta della partecipazione e si rafforza nella condivisione. E procede nel senso della collettività per costruire comunità. 

pare che ci troviamo davanti a una tendenza opposta. Se andiamo a guardare quelle che possiamo chiamare le interpretazioni ultimissime del concetto di rischio, ho l'impressione che non ci aiutino ad andare molto avanti, farci procedere nel senso della condivisione e della partecipazione, anzi ci portano indietro. Agitano ancora i concetti dell'egoismo individualista, fanno riaffiorare le regole del farsi spazio contro tutto e tutti e, molto spesso, anche sulla pelle degli altri (cfr. Taleb 2018). In questi casi l'altro non è più una risorsa in senso comunitario, ma si trasforma in salvagente $o$ in un trampolino. Costruendo così un messaggio esattamente contrario a quello che abbiamo cercato di argomentare in queste riflessioni. Quelli attuali sono percorsi che fanno risorgere la vecchia etimologia (tanto italiana quanto inglese) del termine rischio (risicare, cioè osare), che rimanda al coraggio spavaldo e soggettivo in barba a tutto e a tutti (cfr. Sennet 1999: 80) e richiamano vecchi proverbi in cui il rischio è interpretato come una possibilità per arrivare al successo («chi non risica non rosica»), per farsi strada e farsi spazio (come il molto poco politically correct, «chi non si butta non si corica con le donne belle»).

Queste ultime interpretazioni - americane e di successo - mi hanno fatto tornare in mente una storiella africana con cui concludo. Due amici per tornare al loro villaggio devono attraversare un pezzo di savana. Lungo la strada, nel mezzo della boscaglia, incrociano un leone. I due si immobilizzano. Il leone li guarda e comincia a leccarsi i baffi. I due amici non sanno che fare. Uno si accoscia e comincia a stringere le stringhe delle scarpe. L'altro gli sussurra: «Pensi che se corriamo potremo salvarci?». «Certo!», risponde l'altro. «Se riuscirò a correre più velocemente di te».

\section{BIBLIOGRAFIA}

Abdelmalek S., 2002 La doppia assenza. Dalle illusioni dell'emigrato alle sofferenze dell'immigrato (ed. or. 1999), Cortina, Milano.

Alaszewski A., 2015 «Anthropology and Risk: Insights into Uncertainty, Danger and Blame from Other Cultures. A Review Essay», in Health, Risk \& Society, 17 (3-4): 205-225.

Alliegro E.V., 2018 «Contaminazione ambientale ed elaborazione del rischio sanitario: $\mathrm{i}$ costi dell'incertezza. Una ricerca antropologica sul "gass-ra-doon” nel quartiere “Tamburi” (Taranto)» , in Archivio Antropologico Mediterraneo, 20 (2).

Anderson B., 1996 Comunità immaginate. Origini e diffusione dei nazionalismi (ed. or. 1991), manifestolibri, Roma.

Apolito P., 2014 Ritmi di festa. Corpo, danza, socialità, il Mulino, Bologna.

Appadurai A., 2001, Modernità in polvere (ed. or. 1996), Meltemi, Roma.

Avallone G. (a cura di), 2018 Il sistema di accoglienza in Italia, esperienze, resistenze, segregazione, Orthotes, Nocera I.

Archivio antropologico mediterraneo, Anno XXII, n. 21 (1)| 2019 
Basch L. et al., 1992 Nations Unbound: Transnational Projects, Postcolonial Predicaments, and Deterritorialized Nation-States, Routledge, London.

Bauman Z., 1999 La società dell'incertezza, il Mulino, Bologna.

Beck U., 1994 Riskante Freiheiten. Individualisierung in modernen Gesellschaften, Suhrkamp Verlag, Frankfurt a.M. (trad. it. del primo capitolo in Beck 2000a).

Beck U., 1996 Modernität und Barbarei. Soziologische Zeitdiagnose am Ende des 20. Jahrhunderts, Suhrkamp Verlag, Frankfurt a.M. (trad. it. del sesto capitolo in Beck 2000a).

Beck U., 1997 Kinder der Freiheit, Suhrkamp Verlag, Frankfurt a.M. (trad. it. dei capitoli II-V in Beck 2000a).

Beck U., 2008 Che cos'è la globalizzazione. Rischi e prospettive della società planetaria, (ed. or. 1997), Carocci, Roma.

Beck U., 2000a I rischi della libertà. L'individuo nell'epoca della globalizzazione, il Mulino, Bologna.

Beck U., 2000b La società del rischio. Verso una seconda modernità, (ed. or. 1986), Carocci, Roma (la trad. italiana presenta come postfazione Risk Society Revisited. Theory, Politics, Critiques and Research Programs, in Van Loon J. (ed.), 1999 The Risk Society and Beyond. Critical Issues for Social Theory, Sage, London: 211-229).

Beck U., 2003 Un mondo a rischio, (ed. or. 2002), Einaudi, Torino.

Beck U., 2008 Costruire la propria vita. Quanto costa la realizzazione di sé nella società del rischio, (ed. or. 1977), il Mulino, Bologna.

Benadusi M. (a cura di), 2015 «Antropologia dei disastri. Ricerca, attivismo, applicazione», in Antropologia Pubblica, I (1-2): 33-60.

Benadusi M. et al., 2016 «On the Witness Stand: Environment Crises, Disasters and Social Justice», in Archivio Antropologico Mediterraneo, 18 (2): 17-31.

Boholm A., 2015 Anthropology and Risk, Taylor \& Francis Ltd, London.

Buber M., 1959 Il principio dialogico, (ed. or. 1923), Comunità, Torino.

Castel R., 2004 L'insicurezza sociale. Cosa significa essere protetti?, (ed. or. 2003), Einaudi, Torino.

De Jong S., 2016 «Cultural Brokers in Post-Colonial Migration Regimes», in Dhawan N. at al. (eds.), Negotiating Normativity, Springer, Cham: 45-59.

de Martino E., 1964 Il problema della fine del mondo, in Pietro Prini (a cura di), Il mondo di domani, Edizioni Abete, Roma: 225-231, poi in De Matteis S., 2016 Il leone che cancella con la coda le tracce. L'itinerario intellettuale di Ernesto de Martino, Edizioni d'If, Napoli: 293-296.

De Matteis S., 1991 Lo specchio della vita. Napoli: antropologia della città del teatro, il Mulino, Bologna.

De Matteis S., 2017 Le false libertà. Verso la postglobalizzazione, Meltemi, Milano.

Dell'Acqua P., D’Autilia S., 2013 «Abbandonare quei luoghi, abitare le soglie», in Rivista Italiana di Medicina Legale, 3: 1356-1375.

Dell'Acqua P., D'Autilia S., 2016 «Il ladro di biciclette e il pugile assassino», aut aut, 320: 39-59.

Diamond J., 2013 Il mondo fino a ieri. Che cosa possiamo imparare dalle società tradizionali?, (ed. or. 2012), Einaudi, Torino.

Douglas M., 1991 Come percepiamo il pericolo. Antropologia del rischio, (ed. or. 1986), Feltrinelli, Milano. 
Douglas M., 1996 Rischio e colpa, (ed. or. 1992), il Mulino, Bologna.

Douglas M., Wildavsky A., 1982 Risk and Culture: An Essay on the Selection of Environmental and Technological Dangers, California University Press, Berkeley.

Eriksen T.H., 2017 Fuori controllo. Un'antropologia del cambiamento accelerato, (ed. or. 2016), Einaudi, Torino.

Evans-Pritchard E., 1976 Stregoneria, oracoli e magia tra gli Azande, (ed. or. 1937), Franco Angeli, Milano.

Gellner E., 1986 Nazioni e nazionalismo, (ed. or. 1984), Editori Riuniti, Roma.

Giddens A., 1994 Le conseguenze della modernità. Fiducia e rischio, sicurezza e pericolo, (ed. or. 1990), il Mulino, Bologna.

Gluckman M., 2013 [1955] Order and Rebellion in Tribal Africa, vol. IV, Routledge, London.

Grassi P., 2018 «L'angosciosa resistenza: decostruire la categoria dell'“abbandono istituzionale" nel quartiere di edilizia popolare di San Siro (Milano)», in Archivio Antropologico Mediterraneo, 20 (2).

Hannerz, U., 1996 Transnational Connections, London, Routledge.

Harthorn B.H., Oaks L. (eds), 2003 Risk, Culture and Health Inequality. Shifting Perceptions of Danger and Blame, Praeger, London.

Hoffman S.M., Oliver-Smith A. (eds), 2002 Catastrophe and Culture. The Anthropology of Disaster, School of American Research Press, Santa Fe-Oxford.

Hüsken U., Neubert F. (eds), 2012 Negotiating Rites, Oxford University Press, Oxford.

James D., 2011 «The Return of the Broker: Consensus, Hierarchy, and Choice in South African Land Reform», in Journal of the Royal Anthropological Institute, 17 (2): 318-338.

Le Breton D., 2017 Sociologia del rischio, (ed. or. 1995), Mimesis, Milano.

Quammen D., 2014, Spillover. L'evoluzione delle pandemie, (ed. or. 2012), Adelphi, Milano.

Raeymaekers T., 2014 Violent Capitalism and Hybrid Identity in the Eastern Congo. Power to the Margins, Cambridge University Press, Cambridge.

Russell M., Gruber M., 1987 «Risk Assessment in Environmental Policy-Making», in Science, 236: 286-290.

Schmidt D., Palutan G., 2018 «Cibo e rifugiati nella città capitolina, tra pratiche di emergenza e tentativi di agentività», in Archivio Antropologico Mediterraneo, 20 (2).

Sennet R., 1999 L'uomo flessibile. Le conseguenze del nuovo capitalismo sulla vita personale, (ed. or. 1998), Feltrinelli, Milano.

Slovic P., 1987 «Perception of Risk», in Science, 236: 280-285.

Taleb N.N., 2018 Rischiare grosso, (ed. or. 2017), Il Saggiatore, Milano.

Tarabusi F., 2014 «Politiche dell'accoglienza, pratiche della differenza. Servizi e migrazioni sotto la lente delle politiche pubbliche», in Archivio Antropologico Mediterraneo, XVI (1): 45-61.

Trickett E.J. et al., 2010 «Towards an Ecology of the Culture Broker Role: Past Work and Future Directions», in Medi Azioni, 10: 88-104.

Turner V., 1993 Antropologia della performance, (ed. or. 1986), a cura di Stefano De Matteis, il Mulino, Bologna. 
Turner V., 2013 Antropologia dell'esperienza, a cura di Stefano De Matteis, il Mulino, Bologna.

Wilson R., Crouch E.A., 1987 «Risk Assessment and Comparisons: An Introduction», in Science, 236: 267-270.

Zinn J.O., 2015 «Towards a Better Understanding of Risk Taking: Key Concepts, Dimension and Perspectives», in Health, Risk \& Society, 17 (2): 99-144.

\section{NOTE}

1. La bibliografia sulla nozione di rischio è ampia e fin troppo variegata. Questo è dovuto al fatto che, come abbiamo cercato di argomentare nella prima parte, tale categoria, nella sua ampiezza onnicomprensiva, include tante varianti anche molto diverse tra loro: si va dalla generale percezione del rischio individuale a quelle relative alle malattie "ambientali", al rischio sanitario (dall'Aids di qualche decennio fa alle diffusione delle epidemie, dalla mucca pazza a Ebola), agli eventi naturali come terremoti, alluvioni, frane, ecc. Per un'ampia carrellata sulla varietà dei temi e dei riferimenti rimandiamo ad alcuni articoli apparsi su «Science»: mi riferisco ai saggi di Wilson R., Crouch E.A., 1987 Risk assessment and comparisons: an introduction, 236: 267-270, Slovic P., Perception of risk, 236: 280-285, Russell M., Gruber M., 1987 Risk assessment in environmental policymaking, 236: 286-290.

Per ulteriori approfondimenti in molte delle direzioni indicate segnaliamo in particolare la rivista «Health, Risk \& Society» (Routledge) di cui proponiamo una selezione essenziale: Zinn J. O., 2015 Towards a better understanding of risk taking key concepts, dimension and perspectives, 17 (2): 99-144 e, sulla stessa rivista, vedi anche Alaszewski A., 2015 Anthropology and risk: insights into uncertainty, danger and blame from other cultures. A review essay, 17 (3-4): 205-225.

Anche Archivio Antropologico Mediterraneo ha dedicato numerose riflessioni sul tema. In riferimento a quanto abbiamo argomentato in queste pagine elenco i rimandi necessari: Tarabusi F., 2014 Politiche dell'accoglienza, pratiche della differenza. Servizi e migrazioni sotto la lente delle politiche pubbliche, 16 (1): 45-61; Benadusi M. et al., 2016 «On the Witness Stand: Environment Crises, Disasters and Social Justice», 18 (2); Grassi P., 2018 «L'angosciosa resistenza: decostruire la categoria dell'“abbandono istituzionale" nel quartiere di edilizia popolare di San Siro (Milano)», in 20 (2); Schmidt D., Palutan G., 2018 «Cibo e rifugiati nella città capitolina, tra pratiche di emergenza e tentativi di agentività», in 20 (2); Alliegro E.V., 2018 «Contaminazione ambientale ed elaborazione del rischio sanitario: i costi dell'incertezza. Una ricerca antropologica sul "gass-radoon" nel quartiere "Tamburi" (Taranto)», in 20 (2).

Nell'elaborare la figura del performer come broker, cercando di ampliare e, soprattutto, di rafforzare "culturalmente" la funzione del semplice mediatore o intermediario, abbiamo segnalato alcuni passaggi necessari che da Gluckman 2013 [1955] Order and Rebellion in Tribal Africa (vol. IV, Routledge, London), conducono alle ultime riflessioni in merito: Trickett E.J. et al., 2010 «Towards an Ecology of the Culture Broker Role: Past Work and Future Directions», in Medi Azioni, 10: 88-104; James D., 2011 «The Return of the Broker: Consensus, Hierarchy, and Choice in South African Land Reform», in Journal of the Royal Anthropological Institute, 17 (2): 318-338; Raeymaekers T., 2014 Violent Capitalism and Hybrid Identity in the Eastern Congo. Power to the Margins, Cambridge University Press, Cambridge; De Jong S., 2016 «Cultural Brokers in Post-Colonial Migration Regimes», in Dhawan N. at al. (eds), Negotiating Normativity, Springer, Cham: 45-59. Si tratta di testi che costruiscono un possibile orizzonte critico e argomentativo aperto e polifonico sul tema centrale del performer come "attivista" al confine di culture diverse. Per il tema dell'antropologia del rischio, oltre ai testi citati nel corso del saggio, il lettore troverà qui ulteriori riferimenti, approfondimenti e ampliamenti.

2. Ho affrontato dettagliatamente questi temi in De Matteis 2017. 
3. Si tratta di una corrente degli anni Novanta che ha intrecciato la caduta dello stato nazione per effetto della globalizzazione. Su questi temi cfr.: Anderson 1991, Hannerz 1996, Basch et al. 1992, Appadurai 2001.

4. Basti guardare la ricaduta della condizione postmoderna nella prospettiva filosofica e antropologica di autori come Gianni Vattimo per rendersene conto.

5. Il riferimento è (ovviamente) a Edward Evans-Pritchard 1937.

6. Per rimanere al solo versante italiano, basti pensare a molte delle opere di Ernesto de Martino.

7. «Cosa hanno in comune avvenimenti tanto diversi quali il disastro di Cernobyl, gli sconvolgimenti climatici, il dibattito in materia di manipolazione genetica, la crisi finanziaria dei paesi asiatici e la minaccia attuale degli attentati terroristici? Rivelano tutti una discrepanza tra la lingua e la realtà, una discrepanza che io chiamo "società mondiale del rischio"», così Beck 2003: 7.

8. Per una rassegna dei vari usi del termine, rimandiamo a Le Breton 2017.

9. Beck «usa la parola "rischio" con il significato di pericolo» sostiene Mary Douglas (1996: 49) e continua: «I rischi, nel senso in cui Beck usa il termine, sono sviluppi scientifici, tecnici o sociali incontrollabili, nati molto tempo prima che i loro effetti collaterali o le loro conseguenze di lungo termine fossero noti» (ivi: 49-50).

10. Abbiamo altri casi in cui si fa riferimento a "forze" esterne o oscure: sempre a Milano, estate del 2010: Oleg Fedchenko, un ucraino di 25 anni, abita con la madre, fa lavori saltuari, frequenta la palestra come pugile e fa uso di sostanze stupefacenti; è stato lasciato dalla fidanzata ed è in crisi di astinenza da cocaina; una mattina esce per vendicarsi, incontra una donna filippina e l'aggredisce uccidendola. In carcere dichiarerà che nel volto della vittima - la prima donna incontrata per caso - aveva colto i tratti del diavolo. Cfr. Dell'Acqua, D'Autilia 2017: 39-59.

11. Ho chiesto al Tribunale di Milano di poter esaminare gli atti processuali.

12. La perizia viene eseguita dalla criminologa Isabella Merzagora e dallo psichiatra Ambrogio Pennati, cfr. <ricerca.repubblica.it/repubblica/archivio/repubblica/2013/10/09/kaboboscontera-la-sua-pena-poi-finira.html>.

13. Sul sistema di accoglienza in Italia e i suoi limiti si veda, per esempio, Avallone et al. 2018.

14. Ho analizzato il concetto di tradizione e i suoi usi in De Matteis 1991: 51-90. Sull'esperienza, si veda De Matteis 2017: 191-202.

15. Su questi argomenti cfr. Abdelmalek 2002.

16. Sul performer come broker vedi: Gluckman 2013 [1955], Trickett et al. 2010, James 2011, De Jong 2016, Raeymaekers 2014.

17. Potremmo chiamarlo anche "operatore ritmico", facendo ricorso all'uso che ne fa Apolito (2014: 215-257).

18. Cfr. anche Dell'Acqua, D'Autilia 2013: 1356-1375.

\section{RIASSUNTI}

Vengono qui presi in considerazione i concetti di incertezza e rischio e la loro nuova configurazione nella società globalizzata. Basandosi su un'analisi di situazioni etnografiche radicate nella storia dell'antropologia e sull'interpretazione di un evento criminale commesso in Italia, il testo mira a lavorare sui concetti di rischio e vulnerabilità come categorie sociali, che è possibile affrontare attraverso una serie di pratiche culturali. In questa prospettiva, viene messa 
in evidenza la figura del mediatore come "esecutore" culturale, performer: un attore sociale che organizza la complessità culturale e costruisce modelli di inclusione attraverso la partecipazione. La capacità di mediare la diversità culturale, in questo modo, può trasformare il rischio e la vulnerabilità in una forza trainante per il cambiamento sociale nella società contemporanea.

The paper considers the concepts of uncertainty and risk and their new configuration in the globalized society. Drawing on an analysis of ethnographic situations rooted in the history of anthropology as well as on the interpretation of a criminal event committed in Italy, the work aims at working the concepts of risk and vulnerability as social categories, which is possible to face through a set of cultural practices. In this perspective, the paper emphasizes the figure of the mediator as a cultural "performer": a social actor who organizes cultural complexity and builds patterns of inclusion through social participation. The capacity to mediate cultural diversity may turn risk and vulnerability themselves into driving forces for social change in the contemporary society.

\section{INDICE}

Keywords : risk, fear, vulnerability, performer, performance

Parole chiave : rischio, paura, vulnerabilità, performer, performance

\section{AUTORE}

\section{STEFANO DE MATTEIS}

Dipartimento di Filosofia, Comunicazione, Spettacolo, Università degli Studi di Roma Tre stefano.dematteis@uniroma3.it 